INTERNATIONAL DESIGN CONFERENCE - DESIGN 2018

https://doi.org/10.21278/idc.2018.0203

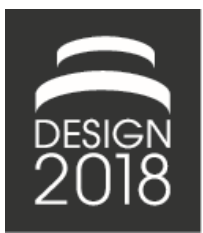

\title{
ROBUST DESIGN FOR MECHATRONIC MACHINE ELEMENTS - HOW ROBUST DESIGN ENABLES THE APPLICATION OF MECHATRONIC SHAFT- HUB CONNECTION
}

\author{
S. Vogel, G. Martin, T. Schirra and E. Kirchner
}

\begin{abstract}
This paper discusses the difficulties caused by production precision for a widespread application of sensorial Mechatronic Machine Elements to expand existing machines or designs to Cyber-PhysicalSystems. The production induced tolerances lead to an undetermined flow of forces in machine elements and prevent a reliable sensing of forces in Mechatronic Machine Elements. A promising approach to overcome these difficulties is Robust Design, which leads to determined mechanical functions that reduce uncertainty of force measurement in Mechatronic Machine Elements.
\end{abstract}

Keywords: robust design, mechatronics, sensor integration, tolerance, cyber physical systems

\section{Introduction}

Current Trends such as Cyber-Physical-Systems (CPS) and Industrial Internet of Things (Industrial-IoT) target the connection and the exchange of data of single machines and even whole production lines, as evolution of mechatronic products (Abramovici, 2015). This supports the automation and optimisation of whole factories and supply chains, by sharing information about the state of the processes. The production rate of a machine can be adjusted to the rate of other machines in the production line in order to reduce unnecessary wear caused by needlessly high production rate. However, in order to share data and information it first needs to be gathered. Applying sensors outside machines or the usage of given data, for example the applied current of an electric motor, can be used to gather relevant information about the process the machine is acting on. Nevertheless, for the determination of the relevant information out of the externally gathered data, calculation models are needed. Depending on the machine and the location the sensor is applied, the modelling takes effort. In the case of either very expensive products, e.g. aviation industry, or high quantities of products, e.g. automotive industry, this effort and cost can be justified. In many other products however, the achievable benefit does not justify a lot of effort, so the designer seeks sensory "Black Boxes" that are easy to implement, do not increase the packaging and deliver reliable and accurate data to simplify the progression to a digitalized world (Martin et al., 2018).

Therefore Mechatronic Machine Elements are presented for integrating sensory functions into Machines. The obstacles to overcome and the potential of Robust Design for the application of Mechatronic Machine Elements shall be discussed using the example of torque sensing shaft-hub connections used for example in Rotary Valves.

Rotary Valves Exist of a sealing element that closes the valve by being rotated by a shaft and reducing the cross section for the volume flow. An actuator provides the necessary torque for turning the shaft and pressing the sealing element in the valve seat. Rotary Valves are used mostly to control volume flows in chemical and power plants and in case of emergencies have to stop the volume flow of partly 
dangerous process media. Because of safety restriction the actuators are mostly pneumatic linear actuators and the rotation is realized either by a simple lever or with a complex transmission that even allows a manual overdrive of the actuator or a manual actuation in case of power failure. The two different functions of the rotary Valve lead to different operations strategies. While controlling the flow of volume the angle of the shaft is measured and the pressure in the actuator is continuously adjusted to achieve the desired angle of the shaft. A closed-loop control for the angle of the shaft is the result. When the valve is closed, an experience-based pressure is set that provides the desired sealing force. This results in an open-loop control of the sealing force. Using developing models (modelling the relation between actuator pressure and mechanic stress in the shaft), the maximum allowed actuator pressure with respect to plastic deformation of the shaft can be calculated.

But because of the large variety of transmissions, actuators and shafts, all of which are often specifically adapted to the application, the designer of this product cannot afford the time-demanding effort to create valid models for all possible variants to predict the stress in the shaft by measuring the pressure. This results in high factors of safety, less performance of the leakage or even in failure of the shafts due to unforeseen disturbance. The effort and uncertainty connected with models would be reduced if a torque sensor in the shaft could be used. However, as pointed out above, there is a significant variety of shaft designs that the torque sensor would have to accommodate. It is therefore proposed to integrate a torquesensing capability in the shaft-hub-connection, which is a standardized interface used in all variants. In this way, a direct torque measurement can be realized in an efficient manner.

\section{Mechatronic Machine Elements}

Mechatronic Machine Elements (MME) sometimes also called Smart Machine Elements, combine sensorial and information processing functions with the easy application which is typical for standardized machine elements. With these combined functions, Mechatronic Machine Elements could close the gap between the trend towards CPS and products that cannot justify the individual development of complex solutions for CPS reaching deep into machines, as pointed out by (Stücheli and Meboldt, 2013). They mentioned the necessary future development in MME, to simplify the development of CPS by using MME, has to decrease the price and the size of MME to lead to plug-and-play solutions. Current research shows that not only the price or size of the MME is an important factor for making them easy to use, but the substitutability (Martin et al., 2018). The latter can be ensured by keeping the standardized mechanical interfaces and the packaging of already existing machine elements.

(Groche and Brenneis, 2014) and also (Schäfer, 2015) developed MME from standard machine elements by adding another function. The load carrying capacity of these machine elements is slightly lowered because of the removal of load carrying material, but the interfaces that connect the machine elements with other parts were kept. On the left hand side of Figure 1 the Smart Feather Key of Schäfer is shown. The Smart Feather Key fits in the standardized keyway in the shaft and can be used as a normal feather key. The right hand side of Figure 1 shows the working principle of the Smart Feather Key. A normal feather key is equipped with strain sensors and by tracking the deformation during load, the torque transferred by the feather key shaft-hub connection can be determined. By changing a simple component as a feather key to a Smart Feather Key, the acting process torque can be measured at any interface where a feather key shaft-hub connection occurs.
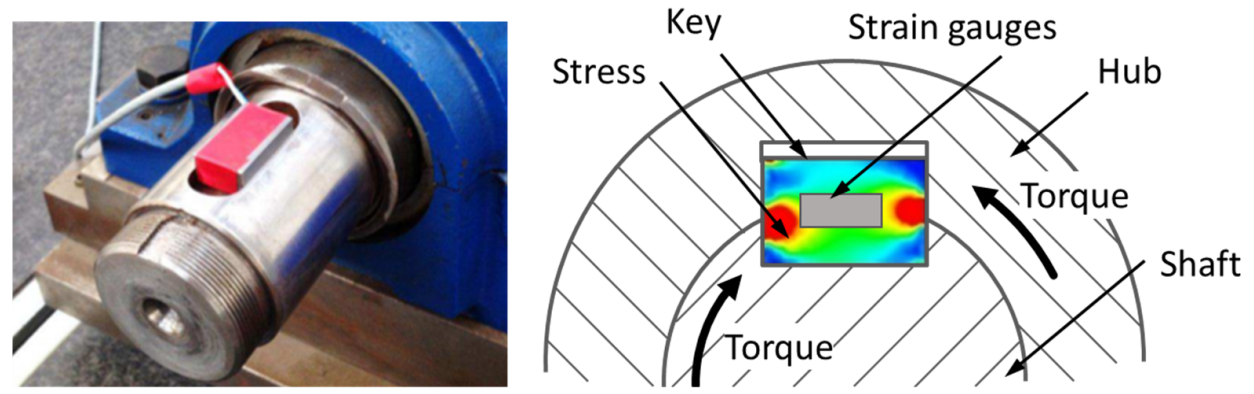

Figure 1. Left: Prototype of mechatronic feather key; Right: Working principle of a mechatronic feather key 
This way, the MME offer the possibility to measure deep inside of machines, at what (Stücheli and Meboldt, 2013) call the "hotspot of action". This enables the designer to measure the interesting values without redesigning the machine (Groche et al., 2015), or developing complex models. This shall be explained using the example of the rotary valve, whose schematic structure is shown in Figure 2 on the left side.

On the right hand side of Figure 2 the components of the rotary valve are analysed and the effects between the components are displayed. The force that acts on the sealing is crucial to the resulting leakage. In this structure the potential of reducing modelling uncertainty and modelling effort through Mechatronic Machine Elements can be seen by evaluating the "distance to the hotspot of action". This is "measured" in terms of transformations and components the interesting value has to pass to reach the possible MME. Each transformation and transfer between the components is associated with modelling effort and disturbance that have to be taken into account or else lead to uncertainty. The colour represents the possible variation of the components in the product variety of the manufacturer. Red components exist in many different variations and the measurement further away from the Hotspot of Action than these components leads to high necessary effort to validate the transformation models. Black components, like the shaft, can differ for different sizes and embodiments of valves, but show a feasible effort to model the most important influences. Green components are standardized and equal in most configurations and offer high potential for developing MME or using already existing solutions.
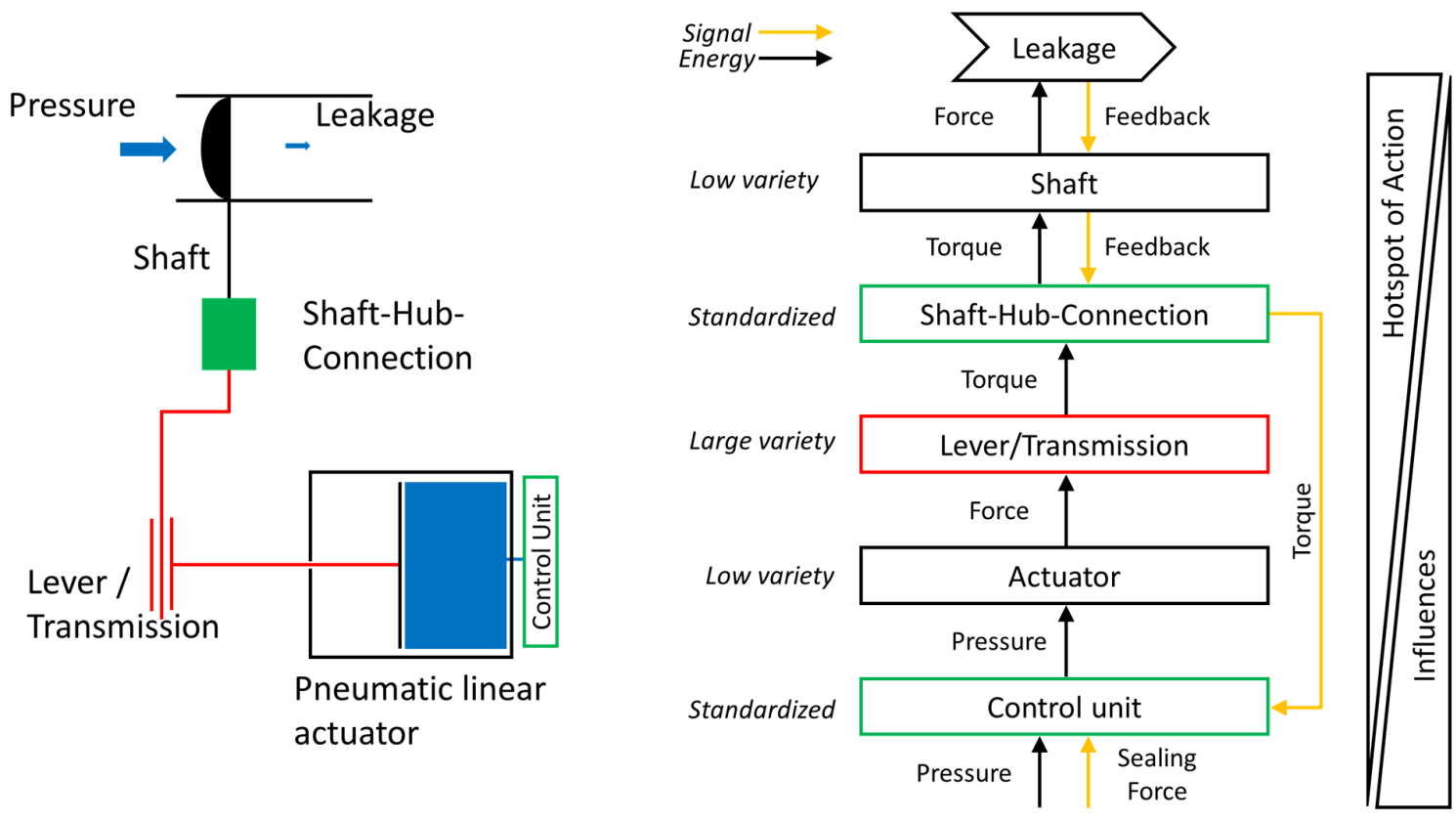

Figure 2. Left: Schematic rotary valve; Right: Modell to determine the sealing torque to reduce leaking

\section{Obstacles to overcome}

MME, like machine elements, exist in different complexities, more complex MME like active magnetic bearings and simpler MME like a sensory key, bolt or shaft couplings. The requirements of these more complex MME differ from the simpler MME and have to be discussed separately. Because complex MME are already established in different applications and the bigger packaging or the higher costs are justified by the unique abilities and the very high performance they can achieve, this paper focuses the obstacle for the application of the simpler MME. This Paper also covers only the issues connected to the mechanical Interfaces. Interactions of the MME with the periphery due to electromagnetic or thermal interfaces introduced by the MME are also still subject of current research, but shall be excluded in this paper. The simpler MME have stricter limits with respect to the packaging or the costs. Therefore the obstacles to overcome are on the one hand the manufacturing of the MME itself - how and what sensor or actuator 
can be applied - and on the other hand the practicability of the MME. The first is a question of manufacturing technology and is not considered in this paper.

The practicability of MME can be ensured by keeping the regular interfaces, packaging and the mounting of the substituted machine elements. But all manufactured parts, due to finite production precision, show geometric variations that can be crucial for the sensory function of an MME.

For example Figure 3 shows the possible distribution of stress in a key for different fitting conditions, namely the largest gap between the parts and the largest interference allowed by the standardized tolerance for feather keys. The stress is representative for a sensed strain by strain gauges. By comparison of the different distributions of stress, shown in Figure 3, it becomes clear that depending on where the strain gauge is applied and which fitting is present, the signal of the strain can differ.
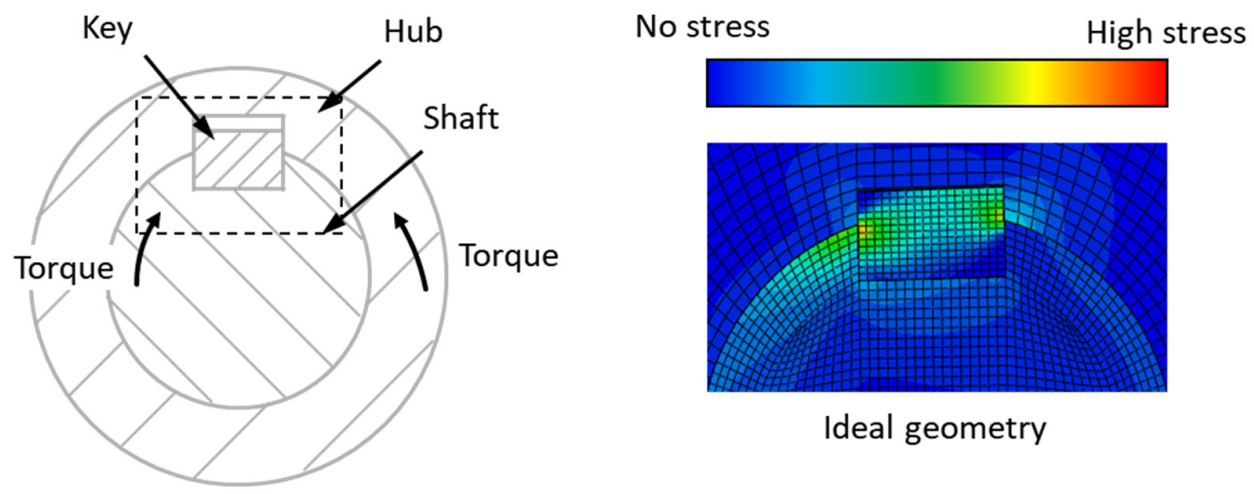

Ideal geometry

\section{Possible geometric Variations:}

Gap between shaft and hub:

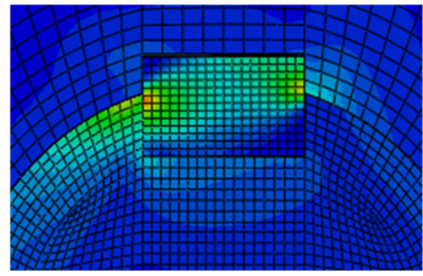

Gap between shaft and key:

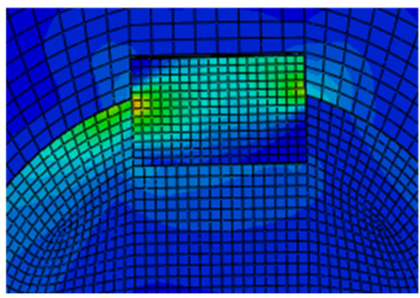

Interference between shaft and hub:

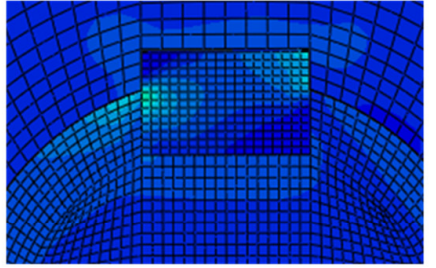

Interference between shaft and key:

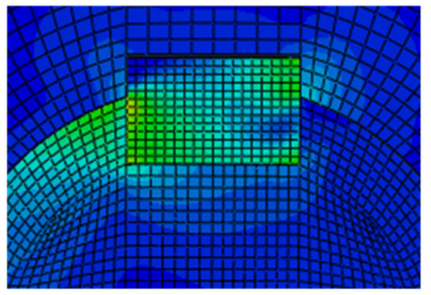

Figure 3. Stress distribution in keys for different fitting condition, with equal torque load; the scale of the stress is for all shown distributions the same ${ }^{1}$

A similar effect of tolerances was shown by (Schork, 2016) for an offset sensing helical beam coupling. (Vogel and Kirchner, 2017) estimated, in a theoretical examination, the influence of the standardized geometrical tolerances for two different types of bolted shaft-hub-connections. The comparison of a friction based and a form fitting shaft-hub connection showed that the strength of the influence differs for the different types. The influence of the geometrical variations on the mechanical function of

\footnotetext{
${ }^{1}$ The Simulations were performed with the commercially available software Abaqus/CAE and show the Mises Equivalent Stress. The shaft was fixed by the function "symmetric-encastre" at the front end. The torque was introduced on the Hub and was evenly distributed at the outer surface of the hub. The interaction of the surfaces is solved as Master-Slave-interaction and a friction coefficient of 0.15 is chosen.
} 
machine elements is mostly known and the standardized tolerances are already a defined trade-off for function and costs (Heling et al., 2016). Therefore raising the production precision without improving the mechanical function of the machine elements is no appropriate solution.

\section{Possible solutions}

The design of MME has to take the tolerances and uncertainties into account and now guidelines have to be developed. A promising approach lies in the principle of Robust Design that helps the product developer to design products that have determined properties for their product lifecycle. For the application of MME the model of the transfer function, developed by (Ulrich and Eppinger, 2008) is suitable to describe the effect of tolerances on the signal. As (Freund et al., 2017) suggested, five types of measures can be identified to increase the robustness of a technical product. Not all of these measures are suited for the application to MME. The possibilities of these measures shall be explained on few examples:

The first type of measures searches for a combination of design parameters that shows minimum sensitivity of the functional performance with respect to variations in the design parameters. This assumes the influence of the tolerance depends on the targeted nominal dimension. In fact the influence of the geometric variances of the products can be nonlinear, as can be seen in Figure 3: The gap fitting and the ideal fitting show almost the same distribution of stress, while the distribution of stress for the ideal fitting and the interference fitting differ clearly. A symmetric form fit shaft-hub connection, like the spline shaft or a feather key connection with more than one feather key shall now be examined to explain the nonlinearity. As shown in Figure 4, on the left side (gap fitting) the torque flow is mostly independent of the fitting tolerance between shaft and hub, as long as they do not intersect. As soon as shaft and hub overlap (interference fitting), as shown in Figure 4 on the right side, a normal force acts on the intersecting surfaces and torque can be transferred by friction between shaft and hub, bypassing the feather keys. The fraction of torque that bypasses the feather keys depends on the actual fit of shaft and hub, which means that the fitting tolerance between shaft and hub now has a significant influence on the significance of the sensor signal. The stress and therefore the strain in the sensing feather key is not representative for the applied load on the shaft-hub connection. A simple measure to reduce the influence of the tolerances is only allowing fittings that lead to tolerances between shaft and hub. The size of the accepted tolerance zone can stay the same, but by shifting the nominal value to smaller shafts und bigger holes in the hubs, an intersection can be avoided. This measure can be realised in already existing systems whose design shall not be changed significantly. Current tolerances are chosen respecting the requirements for mechanical function and the manufacturability. The newly integrated function in MME request additional requirements that lead to a new evaluation of the status quo of the trade-off of functionality and manufacturability.
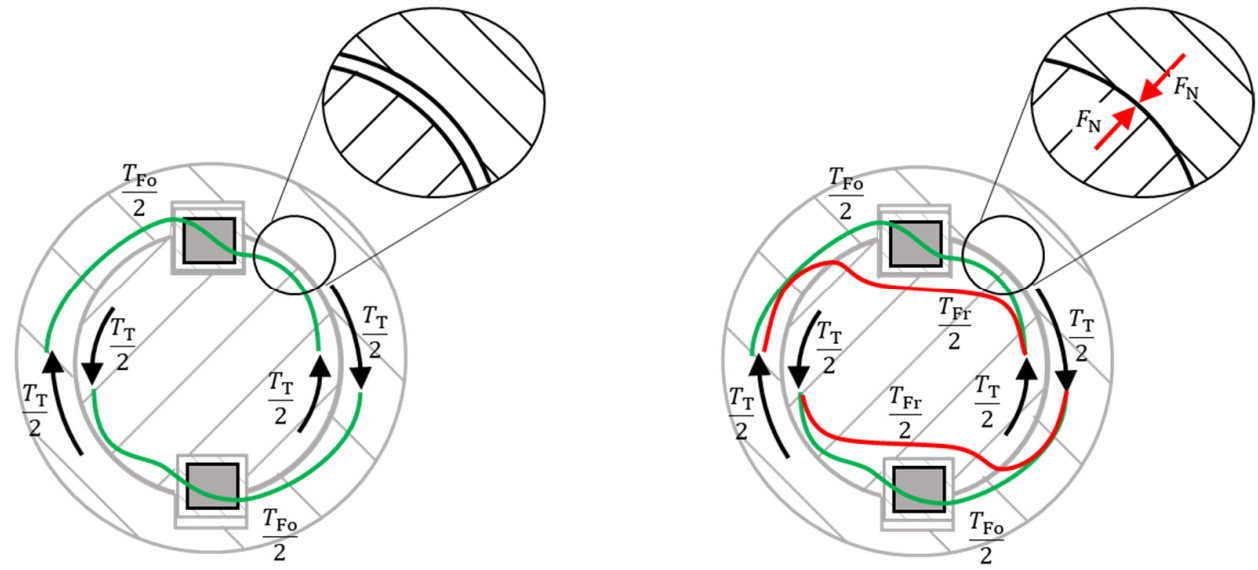

Figure 4. Left: Flow of force without intersection of shaft and hub; Right: Flow of force with intersection shaft and hub

The second type of measures demands less variation of the design parameters to reduce the variation of the functional performance, e.g. through increasing the production precision. This measure is, as previously mentioned, not a useful solution due to rising costs and the requirement of using standardized parts. 
The third type of measures aims to achieve independence between the mechanical functions of the interacting bodies, so they do not influence each other. This measure can be realised by designing the functions explicitly. In many technical designs the force or torque flow is statically over-determined and is split, sometimes due to redundancy. This leads to an unpredictable behaviour of the system and therefore increases the uncertainty of the sensor signal. In mechanical design, functions are often realised by standardized parts or working principles. Some principles deliver the same mechanical function, but less uncertainty for the interpretation of the sensor signal. This shall be discussed on another shaft-hub connection. Figure 5 shows a helical bevel gear connected to the shaft by a spline connection which is secured by a sensory bolt. The shaft-hub connection is overdetermined. The force flow of the torque is transferred by the formfitting faces of the splines, shown in Figure 5 on the right hand side, and by friction between the hub and the shaft collar, for simplicity and to avoid unnecessary confusion not explicitly indicated in the figure. This leads to a higher load capacity, but not completely predictable behaviour, because the split of the torque flow between the paths depends on the operating conditions, e.g. bolt preload, axial operating force and friction coefficient. On the other hand the axial force that is measured by the force sensing bolt to calculate the transferred torque is transferred through the bolt connection, shown in green and blue, and by friction through the formfitting faces, shown in red. This leads to an undetermined splitting of the flow of axial force that is dependent on the tolerance and the current operation condition, e.g. temperature, lubrication and load.

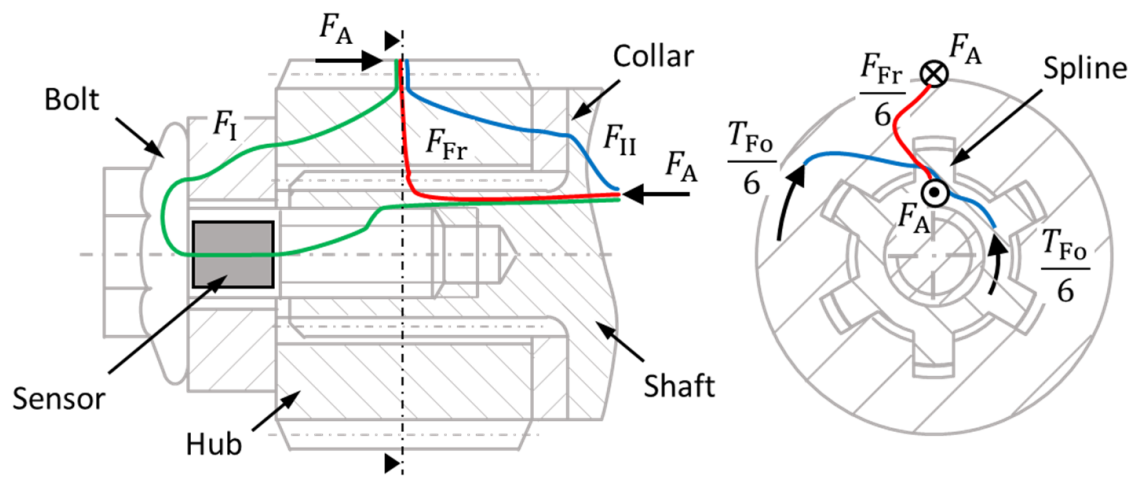
Figure 5. Left: Qualitative splitting of the flow of axial force in an overdetermined shaft-hub connection; Right: Illustration of the idealised transfer of torque for one spline and the resulting transfer of axial force

This results in uncertainty in interpreting the sensor signal: For low force levels, the axial force is only transferred by friction (Figure 5 red path), there is no additional axial load on the bolt and the sensor output is zero. For higher torque levels, there is an additional load on the bolt, enabling the use of the bolt as a torque sensor. Figure 6 shows the expected force on the bolt for the acting torque. The uncertainty of the friction between the splines and the hub leads to an unknown minimum measurable torque. For higher torque values, the splitting ratio of the flow of force between $F_{\mathrm{I}}$ and $F_{\mathrm{II}}$ is, due to the possible variation of the elasticity of the transferring components, also not determinable exactly. This results additionally in difference of gradient of the ideal and expected relation between torque and axial force on the bolt, shown in Figure 6. The influence of the variation in the elasticity is, as shown later in this paper, of minor effect.

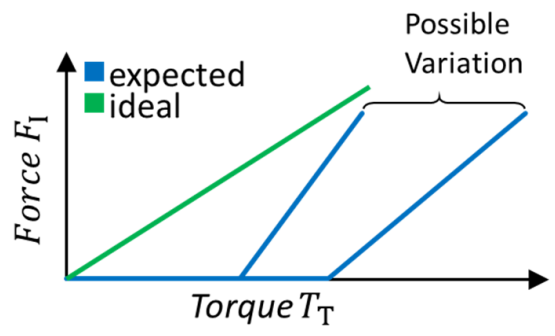

Figure 6. Qualitative expected variation of the measurable force $F_{I}$ due to variation of the friction and the variation of the geometry 
By changing the principle of the shaft hub connection to a friction based shaft hub connection, as shown on the left hand side of Figure 7, the uncertainty can be reduced. The function for torque transfer is realised by only one interface and the transfer of the axial force is realised by only the bolt connection, shown in green and blue. The splitting of the flow of axial force is, due to the known elasticity of the components, determinable. Figure 7 right shows the expected force on the bolt for the applied torque in comparison to the ideal behaviour. The expected splitting of the flow of axial force also shows possible variations due to geometrical variation, but in comparison to the previously shown shaft-hub connection the variation is significant lower.

The measure of using a different, more robust shaft-hub-connection can only be applied when designing a new product or redesigning a product, because a change of the solution principle results in further changes in the machine due to interfaces and different load capacities.
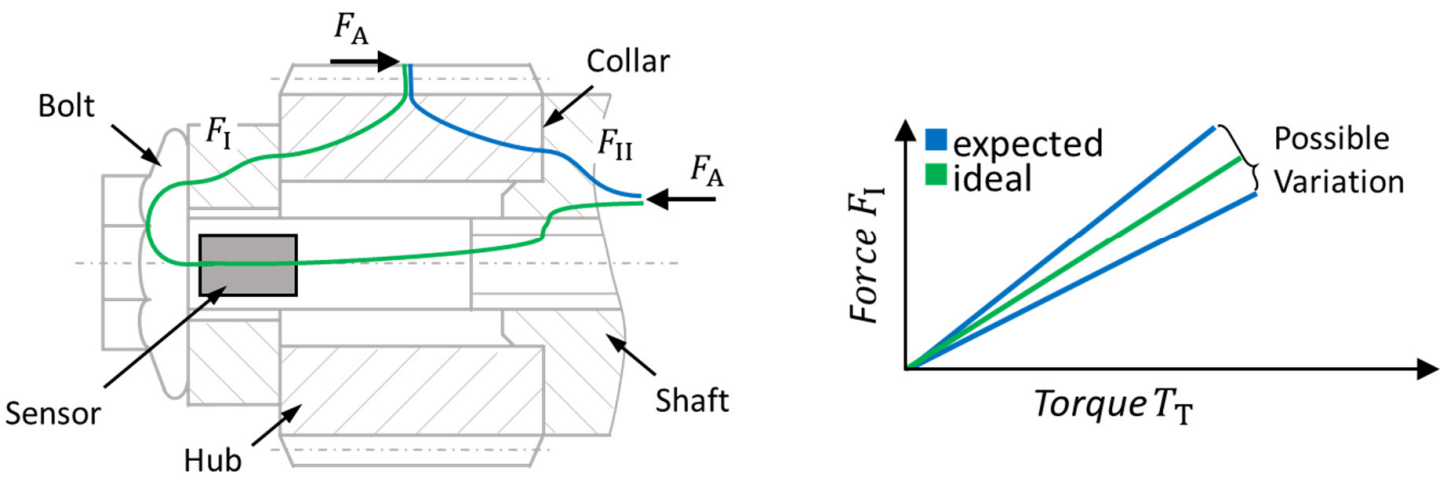

Figure 7. Left: Qualitative splitting of the flow of force in an determined shaft-hub connection; Right: Qualitative influence on the Signal due to variation of the geometry

The fourth type of measures allows more variation of the functional performance, but is no suitable solution for the application of MME, because this would lead to restricting usage conditions or reducing significance of the sensor signal.

The fifth type of measures aims to reduce the potential of errors, but is partly already realised in the description of the third measure: Fewer interacting interfaces lead to less possible disturbing influences and therefore lead to a more robust solution with respect to the sensor function.

\section{Conclusion}

Mechatronic Machine Elements offer the same mechanic function as regular machine elements, but also enable measuring of the load acting on these machine elements. Because the acting loads are representative for the actual state of the machine or even the process the machine is acting on, the benefits are undisputed. But current MME are complex and cost-intensive products that are used for test-rigs or when the higher mechanical performance is mandatory. Simpler MME that sometimes are even composed of only one load carrying component, offer possibilities to measure variables of the machine that cannot be affordably measured the conventional way. The resulting issues implied by the usage of MME are shown, but also the promising results of using robust design measures to overcome the issues caused by the limited production precision of standardized machine elements. Some measures lead to minor changes in the design and can be used to enable existing designs for the usage of MME. Other measures can be used for the search of solution principles and can help to choose a more robust sensor principle for a needed mechanical function. The authors propose for future research the development of guidelines and catalogues for the robust usage of MME or advices for adjustments for the successful usage of MME.

\section{References}

Abramovici, M. (2015), "Smart Products", In: Laperrière L. and Reinhart G. (Eds.), CIRP Encyclopedia of Production Engineering, Springer, Berlin, Heidelberg, pp. 1-5. https://doi.org/10.1007/978-3-642-359507_16785-1 
Freund, T., Würtenberger, J., Lotz, J., Rommel, C. and Kirchner, E. (2017), “Design for robustness - systematic application of design guidelines to control uncertainty", ICED17 21st International Conference on Engineering Design(ICED17), Vol. 4: Design Methods and Tools, Vancouver Canada 21-25 August, pp. 277-286.

Groche, P. and Brenneis, M. (2014), "Manufacturing and use of novel sensoric fasteners for monitoring forming processes", Measurement, Vol. 53 No. 1, pp. 136-144. https://doi.org/10.1007/s11740-014-0576-5

Groche, P., Calmano, S., Felber, T. and Schmitt. S.O. (2015), "Statistical analysis of a model based product property control for sheet bending", Production Engineering, Vol. 9 No. 1, pp. 25-34. https://doi.org/10.1007/s11740-014-0576-5

Heling, B., Aschenbrenner, A., Walter, M.S.J. and Wartzack, S. (2016), “On Connected Tolerances in Statistical Tolerance-Cost-Optimization of Assemblies with Interrelated Dimension Chains”, Procedia CIRP, Vol. 43, pp. 262-267. https://doi.org/10.1016/j.procir.2016.02.031

Martin, G., Schork, S., Vogel, S. and Kirchner, E. (2018), "MME: Potentiale durch Mechatronische Maschinenelemente", Konstruktion, Vol. 01-02, pp. 72-82.

Schäfer, K.R. (2015), Entwicklung und Bewertung von Smart Machine Elements, Master thesis, Technische Universität Darmstadt.

Schork, S. (2016), Schlüsseleigenschaften und Erfolgsfaktoren von Smart Machine Elements, Master-Thesis, Technische Universität Darmstadt.

Stücheli, M. and Meboldt, M. (2013), "Mechatronic Machine Elements: On Their Relevance in Cyber-Physical Systems", Smart Product Engineering: Proceedings of the 23rd CIRP Design Conference, Bochum, Germany, March 11-13, 2013, Springer, Berlin, Heidelberg, pp. 263-272. https://doi.org/10.1007/978-3-642-30817$8 \_26$

Ulrich, K.T. and Eppinger, S.D. (2008), Product design and development, 4th ed., McGraw Hill/Irwin, Boston.

Vogel, S. and Kirchner, E. (2017), "Konstruieren mit Smart Machine Elements - Einfluss von Bauteiltoleranzen und Uneindeutigkeit", Design for X: Beiträge zum 28. DfX-Symposium, October 2017, Tutech Verlag, TuTech Innovation GmbH, Hamburg, pp. 311-323.

Sven Vogel, Research Associate

Technische Universität Darmstadt, Produktentwicklung und Maschinenelemente

Otto-Berndt-Str. 2, 64287 Darmstadt, Germany

Email: vogel@pmd.tu-darmstadt.de 